\title{
Manipulación del Novint Falcon mediante el brazalete de gestos Myo
}

\author{
Manipulation of the Novint Falcon using the Myo Gesture Bracelet
}

\section{Gudiño-Lau Jorge ${ }^{a}$, Alcalá-Rodríguez Janeth ${ }^{b}$, Charre-Ibarra Saida ${ }^{c}$, Durán-Fonseca Miguel ${ }^{d}$, Velez-Diaz Daniel ${ }^{e}$, Jalomo Jaime $^{f}$}

\begin{abstract}
:
This article presents the manipulation of a Novint Falcon three degrees of freedom haptic device through of forearm and hand gesture control bracelet Myo. The communication interface of the Myo wristband and the control of the haptic device are developed in MATLAB Simulink, through libraries and device drivers.

Furthermore shows a linear PID control for the manipulation of the haptic device, having as a reference signal the Myo electromyographic bracelet of a person, it is used to detect forearm and hand gestures. This application is developed so that people with disabilities of the upper limb can manipulate objects.
\end{abstract}

\section{Keywords:}

Novin falcon, Myo bracelet, linear control, simulik, haptic device

\section{Resumen:}

Este artículo presenta la manipulación de un dispositivo háptico Novint Falcon de tres grados de libertad a través de un brazalete Myo de control de gestos de antebrazo y mano. La interfaz de comunicación del brazalalete Myo y el control del dispositivo háptico son desarrollada en Simulink de MATLAB, mediante librerías y controladores propias de los dispositivos.

Además muestra un control lineal PID para la manipulación del dispositivo háptico, teniendo como señal de referencia el brazalete electromiográfico Myo de una persona, se utiliza para detectar los gestos del antebrazo y mano. Esta aplicación está desarrollada para que personas con alguna discapacidad motriz de la extremidad superior pueda manipular objetos.

\section{Palabras Clave:}

Novin falcon, brazalete Myo, control lineal, simulik, dispositivo háptico.

\section{Introducción}

En los últimos años, las investigaciones en el área de la robótica están orientadas a la medicina especialmente al diseño de tecnologías que ayude al diagnóstico y rehabilitación motriz de personas que sufrieron una lesión o degenerativa.

Existen dispositivos hápticos, desde los más sencillos hasta los más complejos, que brindan retroalimentación

\footnotetext{
Autor de Correspondencia, Universidad de Colima, Facultad de Ingeniería y Electromecánica, ORCID: https://orcid.org/0000-0002-0585908X, Email: jglau@ucol.mx

b Universidad de Colima, Facultad de Ingeniería y Electromecánica, ORCID: https://orcid.org/0000-0002-0238-3952, Email: janethalcala@ucol.mx

Universidad de Colima, Facultad de Ingeniería y Electromecánica, ORCID: https://orcid.org/0000-0002-3823-5388, Email: scharre@ucol.mx

d Universidad de Colima, Facultad de Ingeniería y Electromecánica, ORCID: https://orcid.org/ 0000-0002-0780-6192, Email: mduran@ucol.mx

Universidad Autónoma del Estado de Hidalgo, Escuela Superior de Tlahuelilpan, ORCID: https://orcid.org/0000-0001-6488-2960, Email: daniel@uaeh.edu.mx

Instituto Tecnológico de Ciudad Guzmán, ORCID: https://orcid.org/0000-0003-0246-299X, Email: jaimejc@ itcg.edu.mx
} 
de fuerza y que, con ayuda de ambientes virtuales, han sido incorporados a sistemas para la ayuda en mecanoterapia como parte del tratamiento a discapacidades originadas por el ACV (accidente cerebrovascular) principal causa de discapacidad en el mundo [6], diplejía, embolia, entre otros. Hasta el momento estos sistemas han demostrado ser eficaces en el apoyo motivacional a personas jóvenes, y el uso de estas brindan a los especialistas la facilidad de leer datos cuantitativos tales como posición, velocidad, aceleración y fuerza [1].

El Novint Falcon es un dispositivo robótico háptico para escritorio utilizado para simular el tacto en el mundo virtual, permitiendo "sentir" objetos visuales u otras fuerzas físicas, como se muestra en la Figura 1 [2].

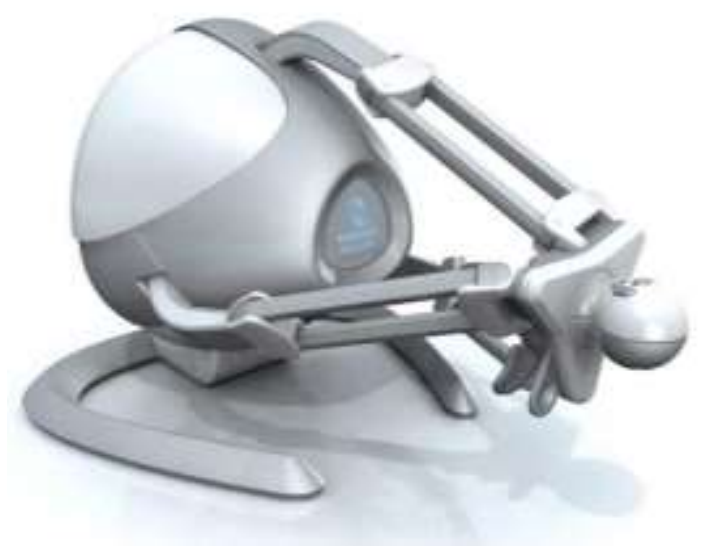

Figura 1. Novint Falcon®.

En este presente proyecto se utiliza como un robot manipulador tipo delta cuya posición en 3 dimensiones será controlada de manera remota, con el objetivo de realizar diagnóstico y rehabilitación de pacientes con alguna lesión en la extremidad superior.

El brazalete Myo es el responsable de recibir las señales electromiográficas generadas por la contracción o relajación mu scular del antebrazo, el girar a la izquierda o derecha, abrir o cerrar la mano, así como los movimientos del brazo, como se observa en la Figura 2. Myo es un brazalete de control de gestos y movimientos que permite controlar dispositivos a distancia mediante movimientos realizados por el usuario.

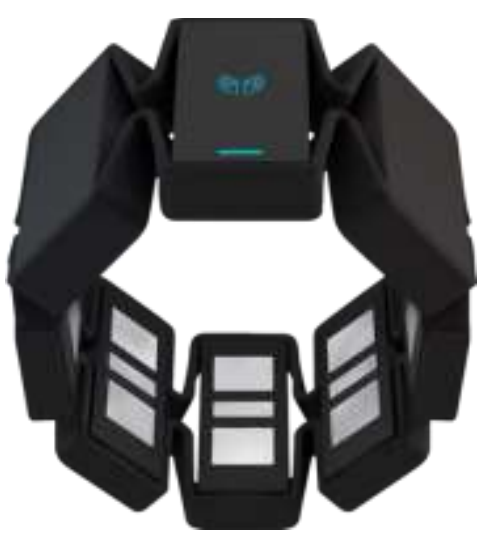

Figura 2. Brazalete Myo.

Myo analiza 8 parámetros: la posición angular del brazo, la fuerza aplicada mediante electromiógrafos, la orientación mediante un giroscopio, la aceleración mediante un acelerómetro, la pose realizada por la mano del usuario (por ejemplo, dedos extendidos, puño, flexión izquierda, flexión derecha, entre otros), indicador de sincronización y un indicador del brazo en el que se encuentra el brazalete (izquierdo o derecho). De todas las anteriores, la posición angular será la única señal que se necesitará adquirir.

\section{Diseño de la interfaz de comunicación}

El programa es realizado en Simulink para interpretar las señales que envía la Myo, se explica a detalle en esta sección. Dicho programa se basa en [5] y en el ejemplo de Simulink dado por la librería háptica.

La primera parte del programa se puede observar en la Figura 2, donde se coloca un bloque de tiempo real (con un bloque terminador para evitar mensajes de advertencia) para sincronizar continuamente el brazalete Myo con Simulink. Además, se tiene el bloque que adquiere, interpreta y envía las señales electromiográficas dadas por Myo. Como se explica, la única señal necesaria para el presente proyecto es la q, el cual expresa los ángulos de rotación del antebrazo del paciente.

Para obtener los ángulos de rotación se realiza una conversión de cuaterniones a ángulos de rotación, como se puede observaren el siguiente bloque del programa en la Figura 4. Dichos ángulos se encuentran en radianes, por lo que se realiza una conversión a grados al multiplicarlo por $180 / \pi$. Se ingresa a un demultiplexor para obtener las 3 señales de manera separada. Como se puede observar, se envia el valor de cada uno de los ángulos en un display. Las 7 señales que no se utilizaron del brazalete, se multiplexaron para enviarse al archivo myo_mat para su procesamiento en Matlab. 

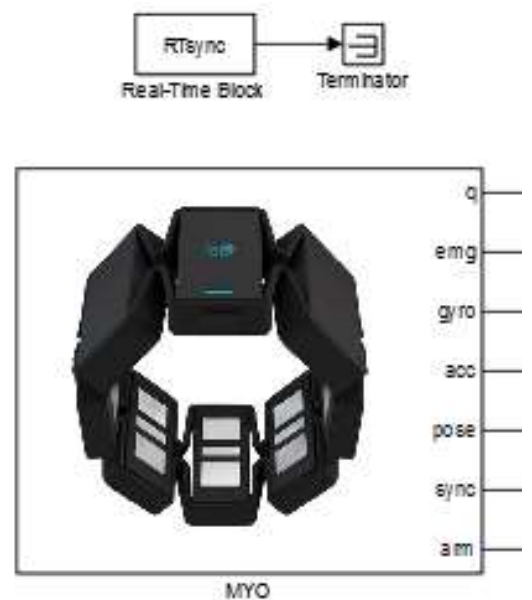

Figura 3. Primera parte del programa: Parámetros del brazalete Myo.

Una vez obtenidos los ángulos de rotación, se ingresaron a una función para obtener la posición final en $x, y, z$, mediante cinemática directa, la cual es la necesaria para mover al Novint Falcon. Cabe aclarar que únicamente se necesitan dos ángulos de rotación debido a que se tomó al brazo como un sistema de 2 grados de libertad, pero fácilmente se puede extender a 3 grados de libertad.

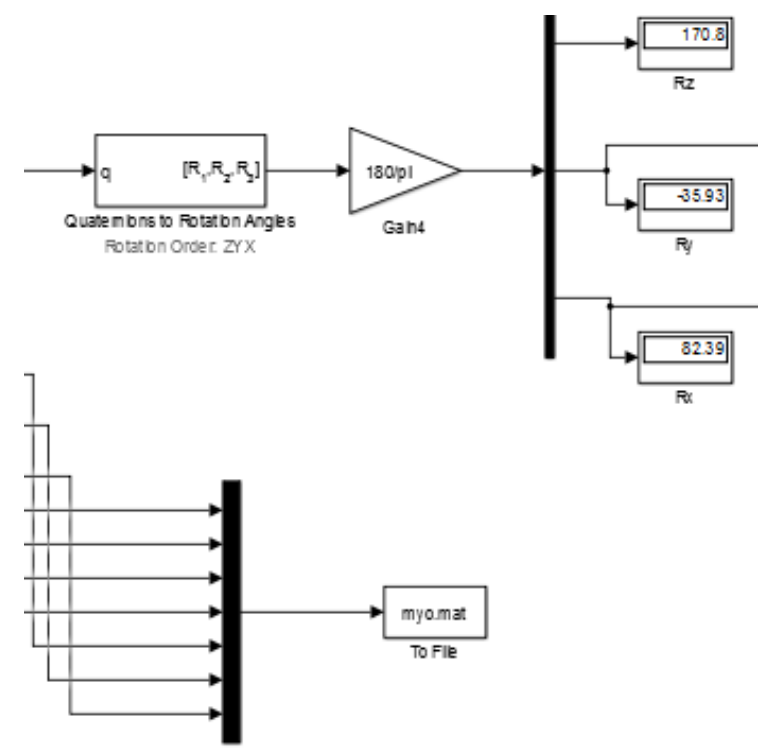

Figura 4. Segunda parte del programa: Obtención de los ángulos de rotación.

Por otra parte, es necesario observar que la cinemática directa corresponde a un brazo con longitud de $90 \mathrm{~cm}$. Aunque dicho valor puede ser modificado de manera sencilla y así, actualizar las matrices homogéneas de la cinemática directa.
La señal de posición en $x, y, z$, es observada mediante un display y se envió al espacio de trabajo de MATLAB, tal como se muestra en la Figura 5.

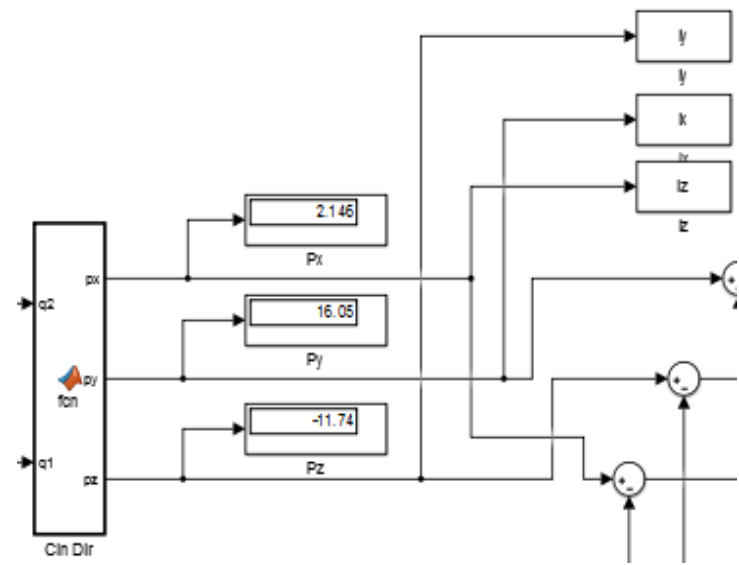

Figura 5. Tercera parte del programa: Obtención de la posición final mediante cinemática directa.

Las posiciones son las entradas del controlador PID (set point). El diagrama del controlador se puede observar en la cuarta parte del programa en la Figura 6. Como se puede analizar, el bloque responsable de enviar las señales o posiciones al Novint Falcon es el Ilamado "PROVA", cuya entrada son las salidas de los controladores PID multiplexados, y su salida, demultiplexada, regresa al controlador como retroalimentación, como se observa en la Figura 6. Se coloca un timer para poder graficar con respecto al tiempo en el espacio de trabajo de MATLAB.

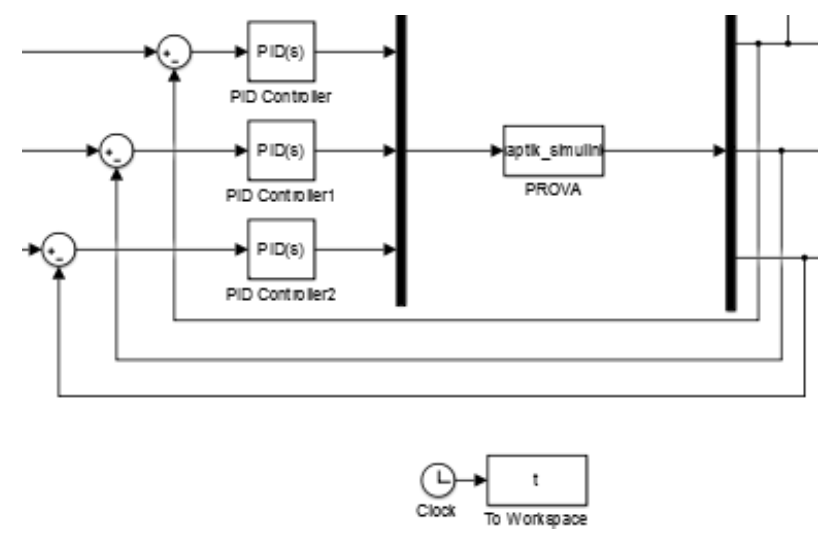

Figura 6. Cuarta parte del programa: Controlador PID.

Por último, la posición final en $X, Y, Z$, ya controlada, se envía al espacio de trabajo de MATLAB y se observa mediante un display, como se ve en la Figura 7. 


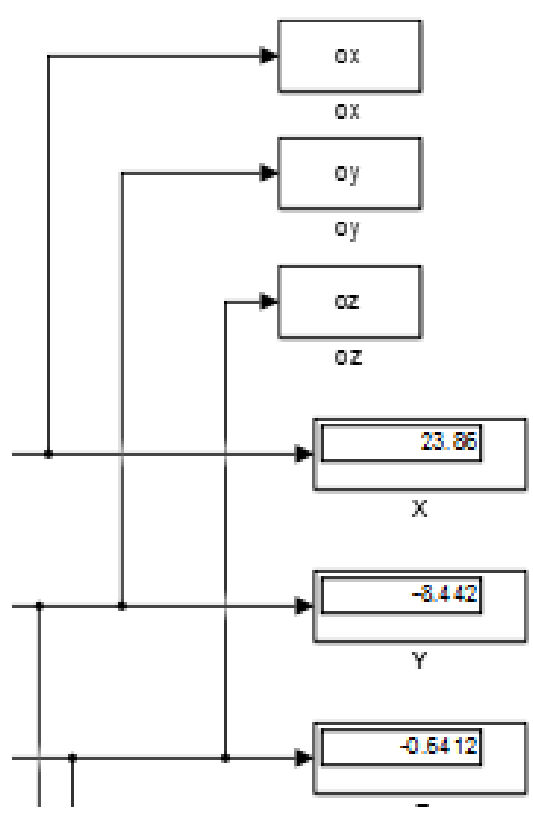

Figura 7. Quinta parte del programa: Envío de la posición final del Novint Falcon®.

\section{RESULTADOS EXPERIMENTALES}

Se conoce la fisiología de los músculos y las señales electromiográficas emitidas por el antebrazo, con la ayuda del brazalete Myo. Además, se identifica las señales cartesianas del antebrazo del paciente.

El Novint Falcon es manipulado correctamente a los movimientos realizados por el usuario con el brazalete. Este principio puede extenderse para controlar una protésis mecánica para que sea manipulada con la señal EMG que envia la Myo o para pacientes con alguna discapacidad motriz, que le permita manipular objetos.

En la Figura 8 se puede observar al brazo del usuario posicionado hacia arriba, el cual es detectado por el brazalete y enviada al Novint Falcon.

El brazo del usuario extendido hacia la derecha es mostrado en la Figura 10, al igual que el Novint Falcon ${ }^{\circledR}$ (esta posición si puede ser alcanzada por el dispositivo de acuerdo a su estructura mecánica).

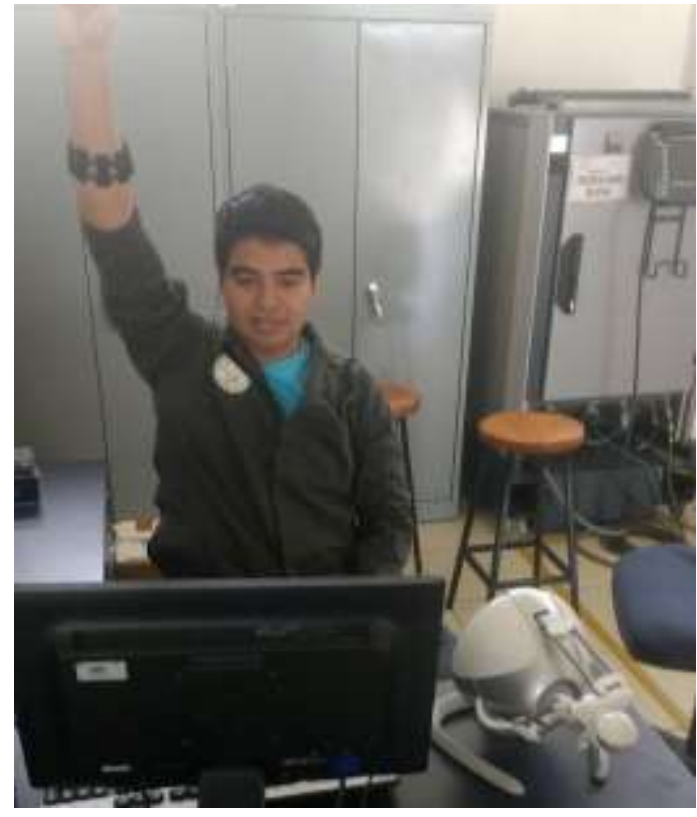

Figura 8. Movimiento hacia arriba del Novint Falcon®.

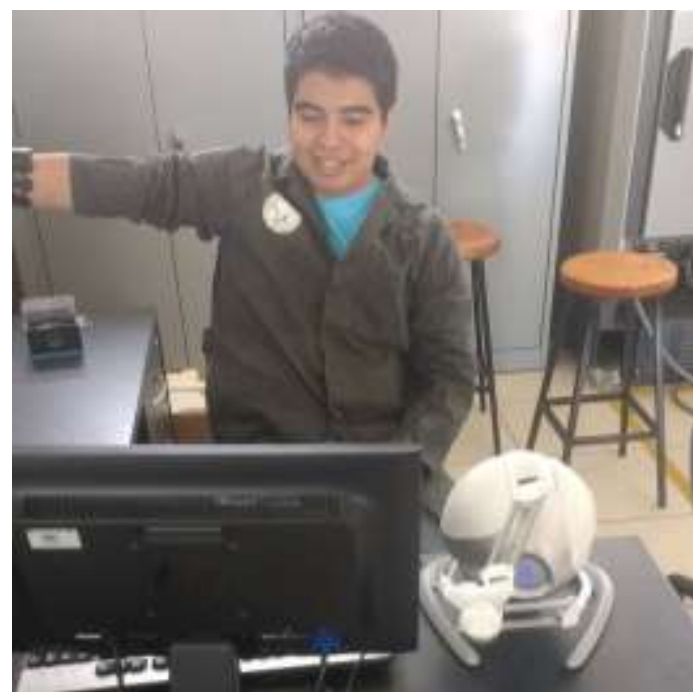

Figura 9. Movimiento hacia la derecha del Novint Falcon®.

Por último, el brazo del paciente fue extendido hacia adelante, y como se observa en la Figura 10, el Novint Falcon respondió a dicho movimiento. 


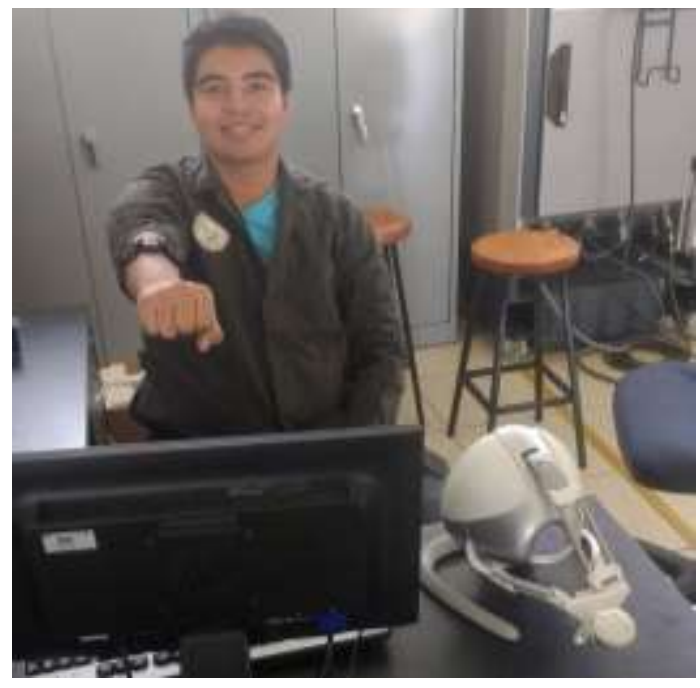

Figura 10. Movimiento hacia arriba del Novint Falcon®.

Se grafica la entrada del controlador (punto de referencia, señal dada por el brazalete Myo, que son las posiciones cartesianas calculadas por la cinemática directa) con respecto al tiempo y se compara con la salida del controlador con respecto al tiempo. En la Figura 11 se puede observar dicha comparación para la coordenada $X$ el cual tiene un buen seguimiento, en la Figura 12 para la coordenada $Y$ y en la Figura 13 para la coordenada $Z$, ambas tienen un buen desempeño.

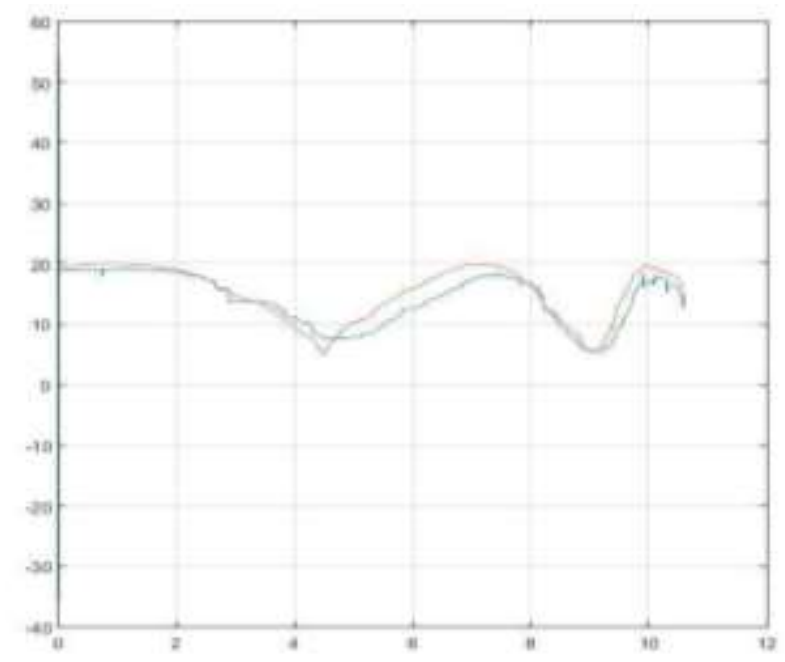

Figura 11. Comparación entre el set point y la salida del controlador con respecto al tiempo en la coordenada $X$.

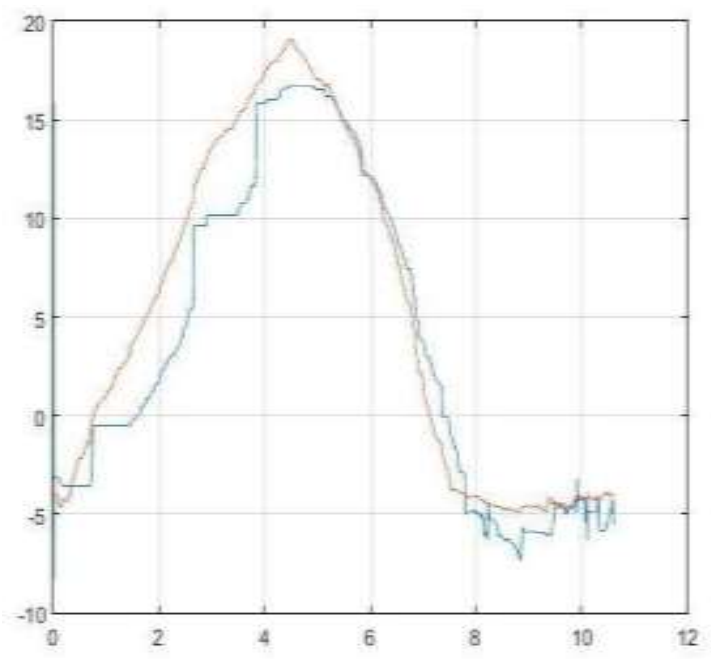

Figura 12. Comparación entre el punto de referencia y la salida del controlador con respecto al tiempo en la coordenada $Y$.

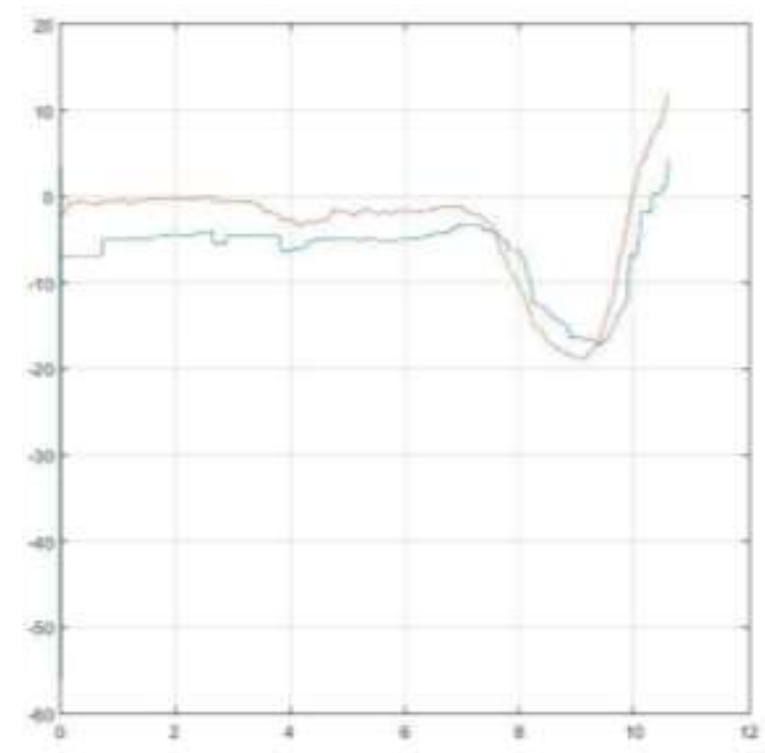

Figura 13. Comparación entre el punto de referencia y la salida del controlador con respecto al tiempo en la coordenada Z.

\section{APLICACIÓN DEL PROYECTO}

Este trabajo tiene como objetivo conocer la señal electromiográfica (EMG) proveniente de los músculos del brazo, y manipular un dispositivo háptico. La adquisición y el registro de la señal EMG de la Myo, es importante para conocer los gestos del antebrazo y aplicarlos a las áreas de investigación como son biomecánicas, fisioterapia, rehabilitación, así como en el control de prótesis para pacientes con amputación de miembros superior o que tengan alguna lesión. 
Con la Myo se adquiere la señal EMG del paciente, identificando el músculo del antebrazo para controlar el dispostivo manipulador como un primer experimento (lo resultados experimentales son mostrados en los capitulos siguientes) y como trabajo futuro desarrollar una protésis mecánica para que sea controlada con la señal EMG o para pacientes con alguna discapacidad motriz del miembro superior.

La idea es adquirir la señal EMG deseada desde la Myo directamente desde los nervios asociados al miembro superior de la mano amputada o dañada. Esto es más delicado ya que requiere de conocer todas las señalesque envía la Myo dado que se requieren el conocimiento de todos los nervios musculares y filtrar la señal para que sea más limpia. Además, se requiere de un control más complejo como un control no lineal que permita manipular la prótesis y obtener la señal la prótesis como forma de retroalimentación al usuario.

\section{CONCLUSIONES}

El control del dispositivo Novint Falcon $\AA$ mediante el brazalete de control de movimientos Myo fue realizado con éxito, sin embargo, se observó que se requieren de gran cantidad de drivers y librerías para poder realizarlo, además de requerimientos de software específicos como Matlab de 32-bits.

Se utiliza un controlador PID debido a su sencillez, sin embargo, se pudo haber aplicado cualquier tipo de controlador debido a que la arquitectura del brazalete es abierta. El error obtenido puede ser mejorado ajustando las ganancias o bien, cambiando de controlador, sin embargo, es un error aceptable. Se recomienda utilizar un control no lineal para manipular una prótesis debido a que son más delicadas.

Este trabajo es el principio y sentará las bases de proyectos futuros de teleoperación de dispositivos manipuladores mediante el brazalete Myo y control prótesis mecánicas accionadas por el propio paciente, dicho movimiento es realizado mediante el miembro superior (antebrazo).

\section{Referencias}

[1] Silva, González y Cruz, Sistema háptico para evaluación y rehabilitación motriz: Resultados experimentales, EAE, 2014.

[2] Virtual Reality, «Virtual Reality Society,» Virtual Reality, [En línea]. Disponible en: https://www.vrs.org.uk/virtual-realitygear/haptic/novint-falcon.html.

[3] Fernando Reyes Cortés., Róbotica. Control de robots manipuladores., Mexico: Alfa omega., 2011.

[4] Ogata, Ingeniería de Control Moderna, Cd. de México: Pretice-Hall, 2010 .

[5] Github, Inc., «S-function for streaming raw data from Myo to Simulink,» Github, 18 Mayo 2015.

[6] Lopez-Larraz E., Ray, Figueiredo TC, Bibian, C., Birbaumer, N., Murguialday R.A. Stroke lesion location influences the decoding of movement intention from EEG. Conf. Proc. IEEE Eng. Med. Biol. Soc. 2017 